\title{
Probing leptophilic dark sectors at electron beam-dump facilities
}

\author{
L. Marsicano, ${ }^{1,2}$ M. Battaglieri, ${ }^{1}$ A. Celentano, ${ }^{1}$ R. De Vita, ${ }^{1}$ and Yi-Ming Zhong ${ }^{3}$ \\ ${ }^{1}$ Istituto Nazionale di Fisica Nucleare, Sezione di Genova, 16146 Genova, Italy \\ ${ }^{2}$ Universitá degli Studi di Genova, 16126 Genova, Italy \\ ${ }^{3}$ Physics Department, Boston University, Boston, Massachusetts 02215, USA
}

(Received 25 October 2018; published 17 December 2018)

\begin{abstract}
Medium-energy electron beam-dump experiments provide an intense source of secondary muons. These particles can be used to search for muon-coupling light dark scalars that may explain the $(g-2)_{\mu}$ anomaly. We applied this idea to the SLAC E137 experiment deriving new exclusion limits and evaluated the expected sensitivity for the planned Jefferson Lab BDX experiment (in case of a null result report). The calculation is based on numerical simulations that include a realistic description of secondary muons generation in the dump, dark scalar production, propagation, and decay, and, finally, the decay product (electrons, positrons, or photons) interaction with the detector. For both experiments, exclusion limits were extended to cover a broader area in the scalar-to-muon coupling vs scalar mass parameter space. This study demonstrates that electron beam-dump experiments have an enhanced sensitivity to new physics in processes that are usually studied using proton beams.
\end{abstract}

DOI: $10.1103 /$ PhysRevD.98.115022

\section{INTRODUCTION}

Fifteen years since it was first proposed [1], the discrepancy between the measured value of the anomalous magnetic moment of muon and its Standard Model (SM) prediction remains unexplained. The so-called " $(g-2)_{\mu}$ anomaly" has provided a strong motivation for light hidden particle searches, opening a window to new physics beyond the SM. Popular candidates such as dark photons and dark Higgs have been tightly scrutinized as a possible explanation for such an anomaly. However, this hypothesis has been excluded by existing measurements (see e.g., [2,3]). Nevertheless, other solutions, such as a new light particle that dominantly couples to muons, remain viable and deserve attention. In this paper, we examine models with a new leptophilic dark scalar, tuned to explain the $(g-2)_{\mu}$ anomaly. We employ a simplified model framework, with the effective Lagrangian for the dark scalar field $S$ written as

$$
\mathcal{L} \supset \frac{1}{2}\left(\partial_{\mu} S\right)^{2}-\frac{1}{2} m_{S}^{2} S^{2}-\sum_{\ell=e, \mu, \tau} g_{\ell} S \bar{\ell} \ell
$$

where $S$ is a real scalar field and the coupling between $S$ and the SM leptons, $g_{\ell}$, is restricted to be

$$
\begin{cases}\text { (lepton-specific) } & g_{e}: g_{\mu}: g_{\tau}=m_{e}: m_{\mu}: m_{\tau} \\ \text { (muon-specific) } & g_{\mu} \neq 0, g_{e}=g_{\tau}=0\end{cases}
$$

Published by the American Physical Society under the terms of the Creative Commons Attribution 4.0 International license. Further distribution of this work must maintain attribution to the author(s) and the published article's title, journal citation, and DOI. Funded by SCOAP ${ }^{3}$.
Such an effective Lagrangian may originate from an effective gauge-invariant dimension 5 operator [4-7]:

$$
\frac{c_{i}}{\Lambda} S \bar{L}_{i} H E_{i},
$$

where $H, L$, and $E$ are the SM Higgs doublet, lepton doublet, and lepton singlet, respectively. $\Lambda$ is the new physics scale and $c_{i}$ are the Wilson coefficients for flavor $i$. Although the relative size of $c_{i}$ and the effective coupling $g_{i}$ are a priori undetermined, one may naturally expect that their values are regulated by the principle of minimal flavor violation (MFV) [8] and therefore proportional to the Yukawa couplings $y_{i}$. This leads to the coupling relations dubbed as "leptonspecific." On the other end, one may go beyond the MFV principle and take the alignment hypothesis [7]; i.e., the scalar only couples to a single fermion of SM and both the scalar and the Yukawa interactions are simultaneously diagonalizable in a single basis. As emphasized in [7], although the UV origin of the alignment hypothesis remains mysterious, it could give rise to couplings of $S$ predominantly to one flavor in a technically natural way that suppresses lepton flavor violations. Here, we choose $S$ to be dominantly coupled to muons and we refer to this scenario as "muon-specific."

Given that scalar-to-quark coupling is absent and its electron coupling is suppressed in a lepton-specific model (or absent in a muon-specific model), the effective way to probe the leptophilic dark scalar is via accelerator experiments with muon beams [9]. Such experiments have been proposed at Fermilab (FNAL- $\mu$ [6], M $^{3}$ [10]) and CERN (NA64- $\mu[6,11]$ ). Alternatively, proposed-proton beam experiments such as SeaQuest [12], NA62 [13], or SHiP [14] can also probe the dark scalars via a significant amount 
of secondary muons or photons. In this paper, we propose a new and alternative way to prove such a model, making use of secondary muons generated in electron beam-dump facilities. Such secondary muons have much larger energy and spatial spread in comparison to muon beams originated by protons. Nevertheless, they are abundantly produced and this search can be performed with a minimal upgrade of existing or proposed electron beam-dump experiments. The novel approach proposed in this paper exploits the fact that, when a high-energy electron impinges on a fixed target, a large variety of secondary particles is produced. This significantly extends the number of physics cases accessible to electron beam-dump experiments.

The paper is organized as follows. Section II describes in general the muon scalar production on a fixed target. Section III describes the main properties of electron beam-dump experiments searching for $S$, including secondary muon production; scalar particle emission, propagation and decay; and, finally, decay products detection. Section IV describes the Monte Carlo-based numerical procedure we developed to evaluate the sensitivity of electron beam-dump experiments. Section V presents results for E137 [15] and BDX [16] experiments and, finally, Sec. VI discusses limits obtained by complementary probes relevant for the parameter space explored in this paper. Appendixes A and B report technical details of the Monte Carlo calculation.

\section{II. $S$ PRODUCTION BY MUONS AND SUBSEQUENT DECAY}

The main process responsible for scalar emission by an impinging muon on a fixed target is the so-called "radiative" production, i.e., the reaction $\mu+N \rightarrow \mu+N+S$, with $N$ being a target nucleus. The corresponding Feynman diagrams are reported in Fig. 1. In the case of large muon energy, compared to the muon and to the scalar mass, the corresponding cross section can be computed at first order using the improved Weizsacker-Williams approximation [6]. The differential cross section, integrated over all the emission angles, reads [17]

$$
\frac{d \sigma}{d x} \simeq \frac{g_{\mu}^{2} \alpha^{2}}{12 \pi} \chi \beta_{\mu} \beta_{S} \frac{x^{3}\left[m_{\mu}^{2}\left(3 x^{2}-4 x+4\right)+2 m_{S}^{2}(1-x)\right]}{\left[m_{S}^{2}(1-x)+m_{\mu}^{2} x^{2}\right]^{2}},
$$

where $x$ is the scalar energy to muon energy ratio $E_{S} / E_{\mu}$, $\beta_{\mu}=\sqrt{1-m_{\mu}^{2} / E_{\mu}^{2}} \simeq 1$ is the muon boost factor, $\beta_{S}=$ $\sqrt{1-m_{S}^{2} / E_{S}^{2}}$ is the scalar boost factor, and $\alpha$ is the fine-structure constant. The effective photon flux $\chi$ is

$$
\chi=\int_{t_{\min }}^{t_{\max }} d t \frac{t-t_{\min }}{t^{2}} G_{2}(t),
$$

where $-t$ is the square of the four-momentum transferred from the initial to the final state nucleus, $t_{\min }$ and $t_{\max }$ are the kinematic limits on $t$, and $G_{2}(t)$ is the combined atomic and nuclear form factor (see Appendix A). The energy distribution of the emitted scalar follows directly from the previous expressions. In the case of a light scalar $\left(m_{S} \ll m_{\mu}\right)$, the $x$ distribution is concentrated in the low- $x$ region, with a maximum value at $x_{\max } \simeq 1.4 m_{S} / m_{\mu}$. For a heavy scalar, instead, the outgoing $S$ takes more significant portion of the muon energy and the corresponding $x$ distribution is peaked close to $x_{\text {peak }} \simeq 1-m_{S} / E_{\mu}$. The kinematic of the produced $S$ is strongly peaked in the forward direction, with a typical emission angle

$$
\theta_{S} \simeq \sqrt{\frac{m_{S}^{2}}{E_{\mu}^{2}} \frac{1-\bar{x}}{\bar{x}}+\frac{m_{\mu}^{2}}{E_{\mu}^{2}}},
$$

where $\bar{x}$ is the typical $x$ value discussed before.

Under our simplified dark scalar model [Eq. (1)], a light scalar $S$ only decays to pairs of photons or leptons once the decay channel is kinetically accessible. The total decay width is given by

$$
\begin{aligned}
\Gamma_{S}= & \sum_{\ell=e, \mu, \tau} \frac{g_{\ell}^{2} m_{S}}{8 \pi}\left(1-\frac{4 m_{\ell}^{2}}{m_{S}^{2}}\right)^{3 / 2} \\
& +\frac{\alpha^{2} m_{S}^{3}}{256 \pi^{3}}\left|\sum_{\ell=e, \mu, \tau} \frac{g_{\ell}}{m_{\ell}} F_{1 / 2}\left(\frac{4 m_{\ell}^{2}}{m_{S}^{2}}\right)\right|^{2},
\end{aligned}
$$

where $F_{1 / 2}(\tau)$ is the fermionic loop function for on-shell scalar and photons. It is given by

$F_{1 / 2}(\tau)= \begin{cases}2 \tau\left[1+(1-\tau)\left(\arcsin \tau^{-1 / 2}\right)^{2}\right] & \tau \geq 1, \\ 2 \tau\left[1-\frac{1-\tau}{4}\left(-i \pi+\ln \frac{1+\sqrt{1-\tau}}{1-\sqrt{1-\tau}}\right)^{2}\right] & \tau<1 .\end{cases}$

In this paper, we primarily focus on the $2 m_{e}<m_{S}<$ $2 m_{\mu}$ case, resulting in a dominant decay into an $e^{+} e^{-}$pair

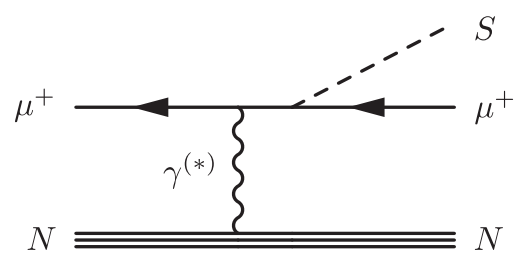

FIG. 1. Feynman diagrams for the radiative dark scalar production by an impinging muon on a nucleus $N$. 
for the lepton-specific model or to a $\gamma \gamma$ pair for the muon-specific case. This is motivated by the fact that the beam-dump experiments here considered have maximum sensitivity to these channels. The corresponding partial widths are

$$
\begin{gathered}
\Gamma_{e^{+} e^{-}}=\frac{g_{e}^{2} m_{S}}{8 \pi}\left(1-\frac{4 m_{e}^{2}}{m_{S}^{2}}\right)^{3 / 2}=\frac{g_{\mu}^{2} m_{e}^{2} m_{S}}{8 \pi m_{\mu}^{2}}\left(1-\frac{4 m_{e}^{2}}{m_{S}^{2}}\right)^{3 / 2}, \\
\Gamma_{\gamma \gamma}=\frac{\alpha^{2} m_{S}^{3}}{256 \pi^{3}}\left|\frac{g_{\mu}}{m_{\mu}} F_{1 / 2}\left(\frac{4 m_{\mu}^{2}}{m_{S}^{2}}\right)\right|^{2},
\end{gathered}
$$

where in the second equality of Eq. (9) we used the identity $g_{e} / m_{e}=g_{\mu} / m_{\mu}$ valid for the lepton-specific model. We note that, for the same values of model parameters $m_{S}$ and $g_{\mu}, \Gamma_{e^{+} e^{-}} \gg \Gamma_{\gamma \gamma}$, resulting in longer-lived $S$ particles for the muon-specific model than for the lepton-specific one.

Finally, we observe that, for the case $m_{S}>2 m_{\mu}, S$ decays always to a $\mu^{+} \mu^{-}$pair, with larger decay width

$$
\Gamma_{\mu^{+} \mu^{-}}=\frac{g_{\mu}^{2} m_{S}}{8 \pi}\left(1-\frac{4 m_{\mu}^{2}}{m_{S}^{2}}\right)^{3 / 2} .
$$

This results in a reduced sensitivity for a typical beamdump experiment, since the large decay width required to cope with the experimental acceptance (see the next section) results to a $g_{\mu}$ value, and hence to a production cross section, too small to have an appreciable production yield.

\section{SEARCHING FOR $S$ WITH SECONDARY MUONS AT ELECTRON BEAM DUMPS}

The typical setup of an electron beam-dump experiment searching for muonphilic scalar particles is shown in Fig. 2. Scalar particles are produced by the primary electron beam impinging on the fixed target through a tertiary process involving secondary muons. These propagate in the target and in the surrounding materials, undergoing energy loss and multiple scattering, and radiatively emit the scalar particles. The resulting $S$ kinematic distribution, including the production vertex, is thus given by the convolution of the muon distribution, altered by energy loss and multiple scattering, and the differential production cross section.

After being produced, $S$ particles propagate straight until decaying to an $e^{+} e^{-}$or $\gamma \gamma$ pair. These particles are measured by a detector placed behind the beam dump. A sizable amount of shielding material is placed between the dump and the detector to range out all other particles produced by the primary beam. Two detection setups are hence possible. In the first case (E137-like), the detector is sensitive to $S$ decay products $\left(e^{+}, e^{-}\right.$, or $\left.\gamma\right)$ produced in a free decay region downstream of the shielding. In the second scenario (BDX-like), the shielding extends up to the

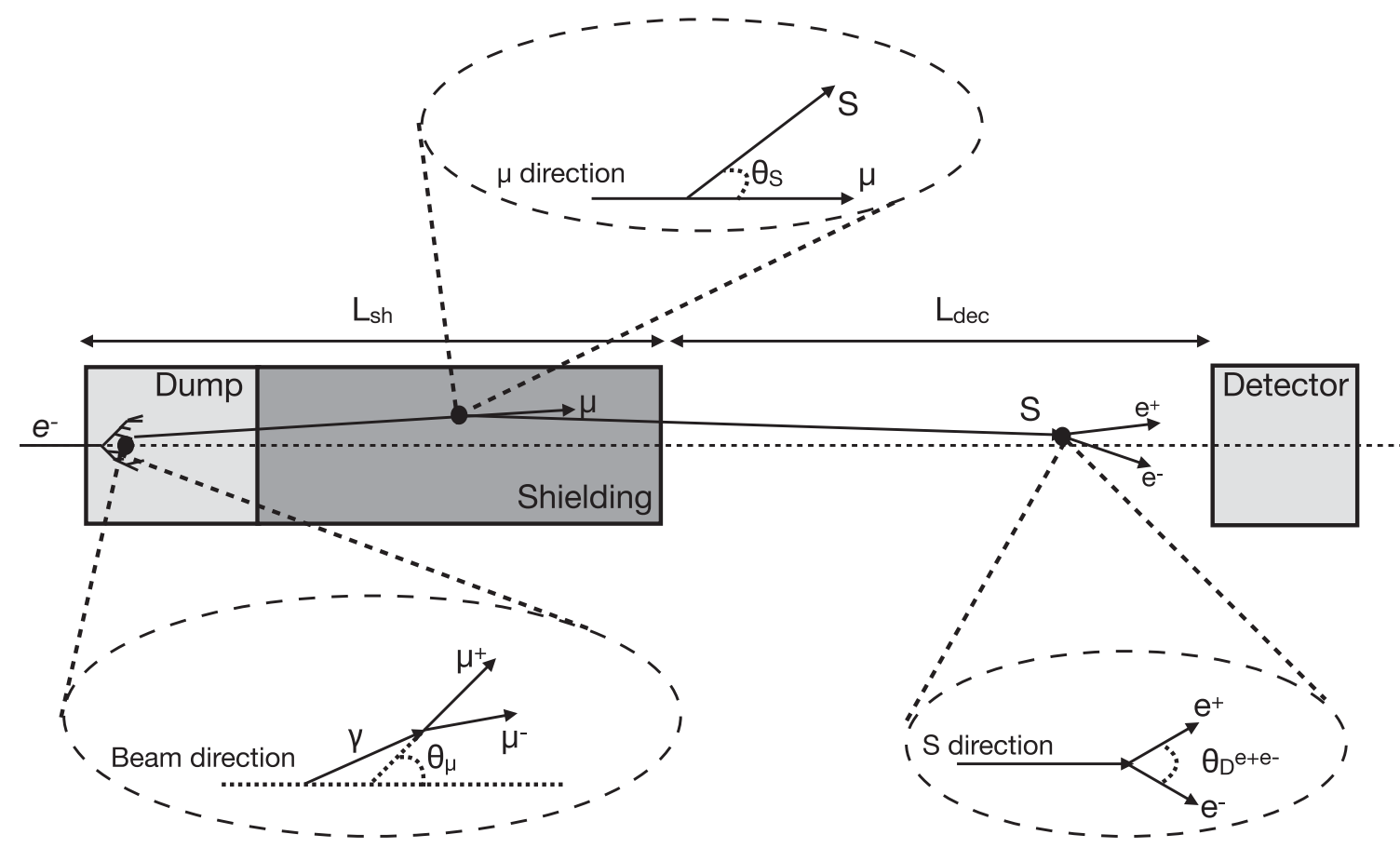

FIG. 2. Typical setup of an "E137-like" electron beam-dump experiment for visible decay $S$ search through secondary muons. $L_{\text {sh }}$ is the total length of target and shielding, while $L_{\mathrm{dec}}$ is the length of the downstream decay region, preceding the detector. The three insets show schematically the production of a $\mu^{+} \mu^{-}$pair by a photon in the dump, the production of $S$ by a secondary muon, and the $S$ decay to an $e^{+} e^{-}$pair. For a "BDX-like" experiment, instead, the shielding extends to the detector position, and the decay region overlaps with the detector volume. 
detector location, and the decay region overlaps with the detector volume.

\section{A. Muons production in $e^{-}$beam dumps}

Production of high-energy muons by an impinging $e^{-}$on a heavy thick target predominantly happens via two different mechanisms, the radiative emission of a $\mu^{+} \mu^{-}$pair and the decay in flight of photoproduced pions and kaons. The contribution of muons produced by the decay at rest of $\pi$ 's and $K$ 's can be neglected due to the very low emission energy, typically lower than the scalar production and detection thresholds.

Muons with energy of the order of the beam energy are mainly produced by pair emission, predominately happening via a two-step process [18]. First, an $e^{-}$radiates a bremsstrahlung photon in a nucleus field, and then the photon produces the $\mu^{+} \mu^{-}$pair in a close-by nucleus. The direct production process through a virtual photon exchange, $e^{-} N \rightarrow e^{-} N \gamma^{*} \rightarrow e^{-} N \mu^{+} \mu^{-}$, is instead negligible, due to the much lower flux of virtual photons. The kinematics of produced muons is strongly peaked in the forward direction, with the typical magnitude of transverse momentum being $p_{\mathrm{T}, \mu} \simeq m_{\mu}$. The pair production on nuclei being a coherent process, the yield of muons produced by the neutral component of the electromagnetic shower through this reaction is almost independent on the target material [see [19], Eq. (9)].

In the low energy range, instead, the dominant production mechanism is the decay in flight of photoproduced $\pi$ 's and $K$ 's. The kinematics of emitted muons is isotropic in the hadron rest frame and shows a forward peak in the laboratory frame whose width depends on the boost factor of the decaying parents. Since $\pi$ and $K$ photoproduction on nuclei scales roughly as the atomic number, the corresponding muon yield shows a significant $1 / Z$ material dependence, $Z$ being the atomic number of the target.

Figure 3 shows the kinetic energy distribution of muons produced by an $11 \mathrm{GeV} e^{-}$beam impinging on a thick aluminum and water target, comparing the full yield to the contribution of photoproduced muons only. This result was obtained from a FLUKA-based $[20,21]$ Monte Carlo simulation, where we implemented the specific target geometry and composition foreseen in the BDX experiment (see Sec. V B). The overall muon yield per electron on target (EOT), in the energy range $0.5-11 \mathrm{GeV}(2-11 \mathrm{GeV})$ is $7.65 \times 10^{-5}$ muons/EOT $\left(0.91 \times 10^{-5}\right.$ muons/EOT $)$. For a primary $e^{-}$beam current of about $10 \mu \mathrm{A}$, this corresponds to a muon rate of $\mathcal{O}\left(10^{9}-10^{10}\right)$ muons/second, depending on the considered energy range. This large flux demonstrates the potential of secondary muons at a multi-GeV electron beamdump facility, in particular when compared to the typical intensity of existing muon beams with similar energies (the Fermilab accelerator complex, for example, can deliver a muon beam of about $10^{7}$ muons/second [22]).

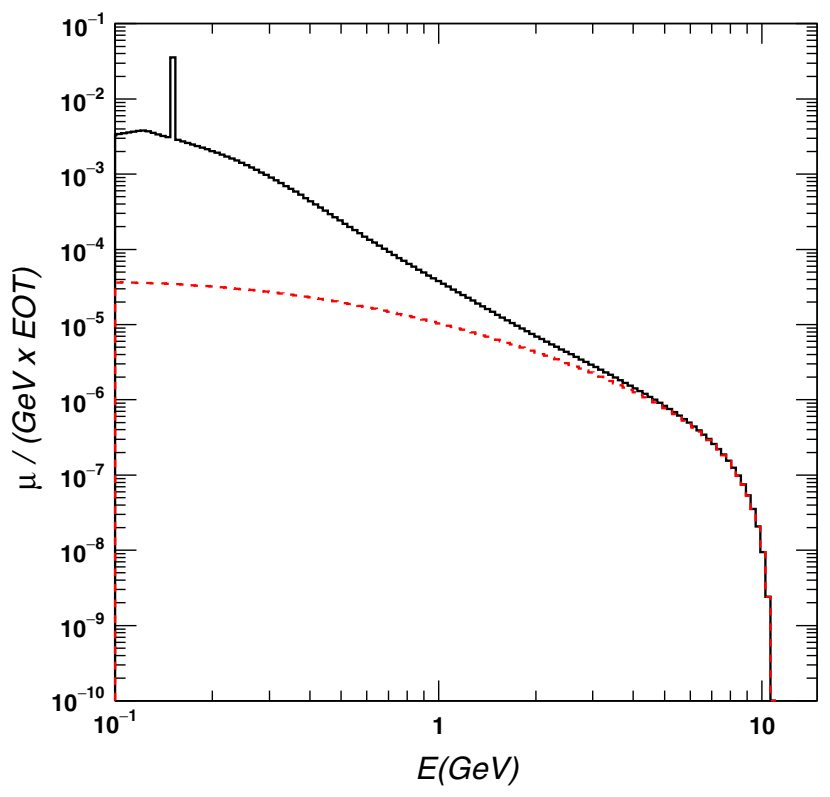

FIG. 3. The differential muons yield per EOT for an $11 \mathrm{GeV} e^{-}$ beam impinging on a thick aluminum and water target, as a function of the muon kinetic energy. The continuous black curve refers to all produced muons, while the dashed red curve refers only to pair-produced muons. The peak in the full distribution at $E=152 \mathrm{MeV}$ is due to the kaon decay-at-rest process, $K \rightarrow \mu \nu_{\mu}$.

Figure 4 shows the kinematic distribution of produced muons, in terms of the emission angle and vertex longitudinal coordinate $z$ (for the latter, $z=0$ corresponds to the target front face.). Higher-energy muons are mostly produced in the forward direction, in the first target radiation lengths, while for lower energies the angular distribution is wider, and particles are produced over the full target length.

\section{B. $S$ production}

Muons produced in the thick target penetrate deeply in it and surrounding materials, losing energy mainly through ionization [24]. While traveling, they may produce a scalar particle through radiative emission on a nucleus.

In the simplest case where the target sizes are larger than the average muon range, and considering an uniform material, the total $S$ production yield is given by

$$
N_{S}=\frac{N_{A}}{A} \rho \int_{E_{\min }}^{E_{0}} d E_{\mu} T_{\mu}\left(E_{\mu}\right) \sigma\left(E_{\mu}\right)
$$

where $A$ and $\rho$, are, respectively, the target material atomic mass and mass density, $E_{0}$ is the primary electron-beam energy, $N_{A}$ is Avogadro's number, $\sigma\left(E_{\mu}\right)$ is the energydependent $S$ production cross section, and $E_{\min } \simeq m_{S}$ is the minimal muon kinetic energy required to produce a scalar with mass $m_{S}$ through radiative emission on a nucleus. Finally, $T_{\mu}\left(E_{\mu}\right)$ is the muon differential track-length distribution in the target, defined as the integral over the target 

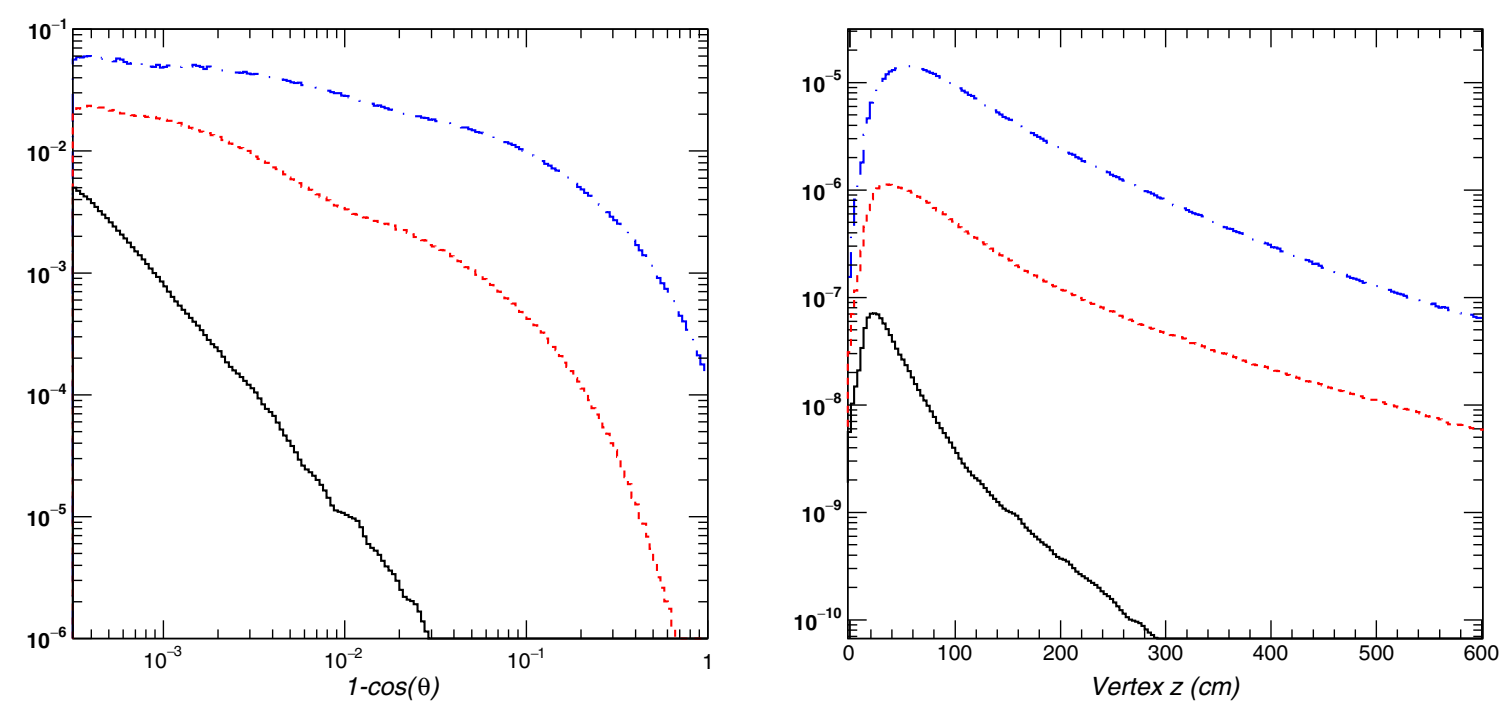

FIG. 4. Kinematic distribution of muons produced in a thick aluminum and water target hit by 11 GeV electrons. Left: Angular distribution; right: longitudinal vertex coordinate distribution (the beam impinges on the thick target at $z=0$ ). The continuous black, dashed red, and dot-dashed blue curves refer, respectively, to 2,1 , and $0.5 \mathrm{GeV}$ muons. The normalization of each curve is proportional to the total muon yield at the corresponding energy.

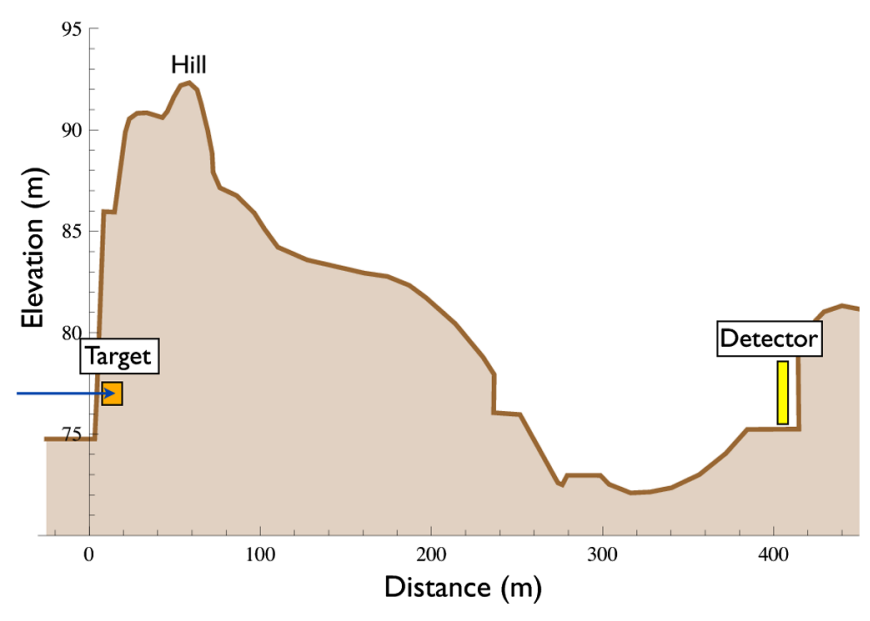

FIG. 5. Layout of the E137 experiment, showing the distance between the beam dump ("target") and the detector. This figure is reproduced from Ref. [23] with authorization from the authors.

volume of the differential muon fluence $\Phi_{\mu}\left(E_{\mu}\right)$, corresponding to the density of particle tracks in the volume [25]. Intuitively, the quantity $T_{\mu}\left(E_{\mu}\right) d E_{\mu}$ represents the total path length in the thick target of muons with kinetic energy in the interval between $E_{\mu}$ and $E_{\mu}+d E_{\mu}$. At the first order, neglecting multiple scattering and considering a constant stopping power $\langle d E / d x\rangle\left(\sim \rho \cdot 2 \mathrm{MeV} \mathrm{g}^{-1} \mathrm{~cm}^{-2}\right), T\left(E_{\mu}\right)$ is given by

$$
T_{\mu}\left(E_{\mu}\right)=\frac{\int_{E_{\mu}}^{E_{0}} n_{\mu}(E) d E}{\langle d E / d x\rangle},
$$

where $n_{\mu}(E)$ is the differential yield of muons in the target. From previous considerations about muon production in the target, and given the shape of $n_{\mu}(E)$, it follows that $T\left(E_{\mu}\right)$ depends on the target material as $(\rho Z)^{-1}$ in the low $E_{\mu}$ region and as $\rho^{-1} \log Z$ at higher muon energy. For a scalar in the mass range here considered most of the contribution to the integral in Eq. (12) comes from the low $E_{\mu}$ region $\left(E_{\mu} \lesssim 1 \mathrm{GeV}\right)$. Therefore, considering the $Z^{2}$ dependence of $\sigma\left(E_{\mu}\right)$ [6], it follows that the total scalar yield depends weakly on the target material.

The kinematic distribution of scalar particles, including the production vertex, replicates the distribution of secondary muons in the beam dump, convolved with the differential production cross section and distorted by the material-dependent mean free path for the $\mu N \rightarrow \mu N S$ process. In particular, the energy distribution of scalar particles (integrated over the full angular range) reads

$$
\frac{d N_{S}}{d E_{S}}=\frac{N_{A}}{A} \rho \int_{E_{\min }}^{E_{0}} d E_{\mu} T\left(E_{\mu}\right) \frac{d \sigma}{d E_{S}}
$$

with $\frac{d \sigma}{d E_{S}}$ given by Eq. (4). From the previous discussion about the shape of $\frac{d \sigma}{d E_{S}}$, it follows that for large $m_{S}$ values, where $\frac{d \sigma}{d E_{S}}$ is peaked at $E_{S} \simeq E_{\mu}, \frac{d N_{S}}{d E_{S}} \propto T_{\mu}\left(E_{S}\right)$. For lower mass values, instead, the broadening induced by $\frac{d \sigma}{d E_{S}}$ is larger. Finally, we note that the shape of $\frac{d N_{S}}{d E_{S}}$ depends significantly on the target material. For example, the most energetic part of the spectrum, for $E_{S} \simeq E_{0}$, is due to the high-energy part of $T\left(E_{\mu}\right)$ and thus exhibits a $Z \log Z$ dependence. 
Previous considerations were derived for a simplified geometry of a large and homogeneous target. In a more realistic case, the target may be nonhomogeneous, and its size may be smaller than the range of muons, requiring a thorough description of surrounding materials. The evaluation of the total $S$ yield and of the corresponding kinematics thus requires one to compute separately $T\left(E_{\mu}\right)$ in each geometry element, by tracking muons in the full experimental setup. For a realistic and accurate evaluation of real experimental setups, we performed this calculation numerically through an ad hoc Monte Carlo simulation, as described in Sec. IV.

\section{Signal yield in the detector}

After being produced, $S$ propagates straight with a differential decay probability per unit path given by

$$
\frac{d P}{d l}=\frac{1}{\lambda} e^{-l / \lambda}
$$

where

$$
\lambda=\frac{E_{S}}{m_{S}} \frac{1}{\Gamma_{S}}
$$

is the energy-dependent $S$ decay length.

Particles from the $S$ decay are emitted on a cone with typical aperture

$$
\theta_{D} \simeq \frac{m_{S}}{E_{S}}
$$

with respect to the $S$ direction. The total signal yield is obtained combining the $S$ angular and vertex distribution with the decay kinematics and projecting the result over the geometrical acceptance of the detector. The latter can be roughly determined as the product of a longitudinal factor $\varepsilon_{L}$ depending on the shielding $L_{\mathrm{sh}}$ and decay region $L_{\mathrm{dec}}$ length and a transverse factor $\varepsilon_{T}$ related to the detector transverse area $A_{T}$ (see Fig. 2). For a single scalar particle produced with momentum $\vec{p}_{S}$ and longitudinal vertex coordinate $z_{S}$, the longitudinal factor reads

$$
P_{L} \sim e^{\left(z_{s}-L_{\mathrm{sh}}\right) / \lambda}\left(1-e^{-L_{\mathrm{dec}} / \lambda}\right) .
$$

The longitudinal acceptance $\varepsilon_{L}$ is obtained convolving $P_{L}$ with the scalar particle kinematic distribution. The transverse factor, instead, reads

$$
\varepsilon_{T} \sim \frac{A_{T}}{\left(\theta_{S}^{\text {rms }}\left(L_{\mathrm{sh}}+L_{\mathrm{dec}}-\bar{z}_{S}\right) \oplus \theta_{D} \bar{L}_{D}\right)^{2}},
$$

with $\theta_{S}^{\text {rms }}$ being the width of the scalar angular distribution, $\theta_{D}$ the typical opening angle between the $e^{+} e^{-}$or $\gamma \gamma$ pair from $S$ decay, $\bar{z}_{S}$ the average $S$ production vertex, and $\bar{L}_{D}$ the average distance between the $S$ decay point and the detector-in the case of a BDX-like setup, with $S$ decaying within the detector volume, $\bar{L}_{D}=0$.

\section{REACH EVALUATION}

To evaluate the BDX reach and to determine the exclusion limit set by the E137 null result for the dark scalar models, one has to determine, for a given combination of the model parameters $\left(m_{S}\right.$ and $\left.g_{\mu}\right)$, the expected number of signal events within the detector acceptance. We performed the calculation of the signal yield numerically, decoupling the evaluation of $S$ particles production from the subsequent propagation and decay as follows.

First, we precomputed the yield of muons produced by the primary electron beam through an optimized simulation using FLUKA 2011.2x.3. In the simulation, we implemented the description of each experimental setup (geometry and materials), including the thick target, the surrounding materials, and the subsequent shielding. We applied different biasing techniques, namely, cross-section enhancement and leading-particle biasing for electromagnetic showers, to enhance forward muon production. The obtained result is a list of produced muons momenta, vertexes and statistical weights, together with the total yield per EOT.

Each muon was then individually tracked through a GEANT4-based simulation [26], implementing the same geometry as implemented in FLUKA. We modified the GEANT4 4.10.02source code to include the new scalar particle $S$ and the radiative $S$ emission process (more details are given in Appendix B). When a scalar particle is produced, the corresponding four-momentum and production vertex are saved to an output file, and the particle is not further tracked. The output of this second step is a list of produced $S$ particles, together with the total normalization.

This result was finally used as input for a custom Monte Carlo code that handles the $S$ propagation and subsequent decay to an $e^{+} e^{-}$or $\gamma \gamma$ pair and computes the experimental acceptance of a detector placed downstream of the dump, $\varepsilon^{\text {det }}$, including geometrical and detection threshold effects.

The overall normalization and the signal yield dependence on model parameters were handled as follows. The first computation step only considers $S$ production, and the corresponding result depends on model parameters through the total number of produced $S$ particles per EOT, $N_{S}^{0} \propto g_{\mu}^{2}$. The corresponding kinematics, instead, does not depend on $g_{\mu}$. Therefore, only the mass of the scalar, selected at the beginning of the simulation, was left as a free parameter while the coupling $g_{\mu}$ was fixed at a conventional value of $g_{\mu}^{0} \equiv 3.87 \times 10^{-4}$. The detection acceptance computed in the second step, instead, depends critically on the $g_{\mu}$ value, affecting the $S$ decay length. Therefore, the evaluation of $\varepsilon^{\text {det }}$ has been performed as a function of this parameter. For a given combination of $m_{S}$ and $g_{\mu}$, the total signal yield in the detector per EOT thus reads 


$$
N_{S}\left(m_{S}, g_{\mu}\right)=N_{S}^{0}\left(m_{S}\right) \cdot \varepsilon^{\operatorname{det}}\left(m_{S}, g_{\mu}\right) \cdot\left(\frac{g_{\mu}}{g_{\mu}^{0}}\right)^{2}
$$

We found this multistep method more effective than performing a single GEANT4 simulation, including both the muon production by the developing electromagnetic shower and the propagation and decay of $S$, since it saves significant computation time in the evaluation of the sensitivity of a beam-dump experiment as a function of the $S$ mass and coupling. Indeed, for a given experimental setup, the muon yield estimation is performed only once. Then, for a given mass value, the $S$ production is evaluated, and only the computation of detector acceptance is repeated for different values of the scalar coupling.

\section{RESULTS}

In this section, we present the exclusion limit in the $m_{S}$ vs $g_{\mu}$ parameters space obtained from the E137 experiment, and the expected sensitivity for the BDX experiment, for both the lepton-specific and the muon-specific models. Results are summarized in Fig. 7, reporting also published limits from other experiments (see the figure caption for further details).

\section{A. E137 exclusion limits}

The E137 experiment searched for long-lived neutral objects produced in the electromagnetic shower initiated by $20 \mathrm{GeV}$ electrons in the SLAC beam dump. Since muons penetrate deeply in the thick target and in the surrounding materials, a precise description of the experimental setup geometry and materials is required. The E137 beam dump was a 720 -cm-long aluminum tank completely filled with water [27,28], with an external diameter of $152 \mathrm{~cm}$. The thickness of the aluminum vessel was $0.9525 \mathrm{~cm}$, a part from the hemispherical front window, with a reduced thickness of $0.475 \mathrm{~cm}$. The vessel radius was $75 \mathrm{~cm}$. The underground hall hosting the dump was made by concrete walls, with a thickness of about $90 \mathrm{~cm}$. The distance between the dump end and the front wall was $160 \mathrm{~cm}$. To protect the concrete wall from low-energy radiation escaping the dump, a 56-cm-long iron block was added. The transverse size of the iron block, determined from the original engineering drawings, was comparable to that of the beam dump.

Scalar particles produced in the thick target would have to penetrate $179 \mathrm{~m}$ of earth shielding and decay in the $204 \mathrm{~m}$ region downstream of the shield, as shown in Fig. 5. We assumed a uniform dirt density of $\rho_{\text {dirt }}=1.7 \mathrm{~g} / \mathrm{cm}^{3}$, a value that seems to us reasonable considering what is reported in Ref. [29] and given the average depth of soil of about $10 \mathrm{~m}$ for the terrain following the underground hall [15]. To evaluate the effect of this parameter on the final result, we repeated the E137 reach computation changing it by $\pm 10 \%$, obtaining a negligible variation of order $\sim 1 \%$.
The E137 detector was an eight-radiation-length electromagnetic calorimeter made by a sandwich of plastic scintillator paddles and iron (or aluminum) converters. Multiwire proportional chambers provided an accurate angular resolution, essential to keep the cosmic background to a negligible level. A total charge of $\sim 30 \mathrm{C}$ was dumped during the live time of the experiment in two slightly different experimental setups: in the first run (accumulated charge $\simeq 10 \mathrm{C}$ ), the detector had a transverse size of $2 \times 3 \mathrm{~m}^{2}$, while in the second run this was $3 \times 3 \mathrm{~m}^{2}$.

The original data analysis searched for axionlike particles decaying in $e^{+} e^{-}$pairs, requiring a deposited energy in the calorimeter larger than $1 \mathrm{GeV}$ with a track pointing to the beam-dump production vertex. The absence of any signal provided stringent limits on axions or photinos.

To derive the E137 exclusion limits on dark scalar production, we used the Monte Carlo-based numerical approach described above. The experimental acceptance was evaluated separately for the two E137 runs and combined with proper weights to account for the different accumulated charges. In the calculation, we employed the same selection cuts used in the original analysis, requiring that the energy of the impinging particle is larger than $1 \mathrm{GeV}$ and that the corresponding angle of impact on the detector, measured with respect to the primary beam axis, is smaller than $30 \mathrm{mrad}$. We found that both particles from $S$ decay hit the detector in a non-negligible fraction of events. In these cases, we applied previous selection cuts respectively considering the sum of the two energies to be greater than $1 \mathrm{GeV}$ and the energy-averaged impinging angle to be less than $30 \mathrm{mrad}$. Based on the null observation reported by E137, we derived the exclusion contour considering a 95\% C.L. upper limit of three events.

\section{B. BDX sensitivity}

$\mathrm{BDX}$ is a planned electron-beam-dump experiment at JLab that will improve the E137 sensitivity by orders of magnitude by using the high-intensity $11 \mathrm{GeV}$ CEBAF beam [30], running for $\sim 1 \mathrm{yr}$ with currents up to $60 \mu \mathrm{A}$, thus collecting $\simeq 10^{22}$ EOT. BDX will make use of the HallA beam dump [31], with the detector placed $20 \mathrm{~m}$ downstream in a new, dedicated experimental hall (see also Fig. 6). In this configuration, the experiment will produce $\sim 10^{17} \mathrm{GeV}$-energy muons in the dump, a total yield much larger than what can be obtained in similar fixed-target efforts employing a primary muon beam [6].

The BDX detector consists of a $50 \times 40 \times 300 \mathrm{~cm}^{3}$ electromagnetic calorimeter made by $800 \mathrm{CsI}(\mathrm{Tl})$ scintillating crystals, surrounded by two active veto layers made by plastic scintillator for cosmic backgrounds rejection. An iron and concrete shielding layer will be installed between the existing Hall-A beam-dump vault and the detector hall to range out all other particles produced in the target (except neutrinos). 


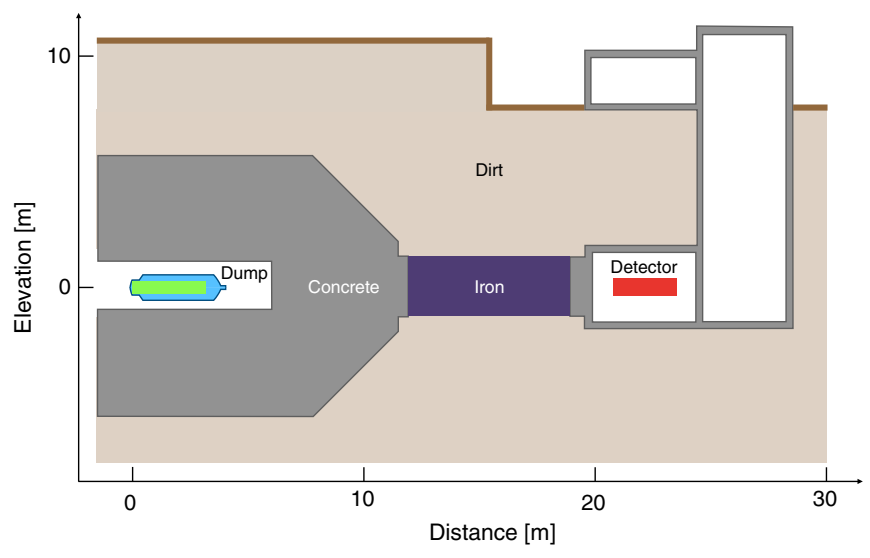

FIG. 6. Implementation of the BDX experimental setup in FLUKA. From left to right, the Hall-A aluminum-water beam dump (blue-green), the concrete walls (gray), the iron shielding (purple), and the detector (red) in the new experimental hall. The two scales report distances between elements (in meters). The detector is located approximately $20 \mathrm{~m}$ downstream from the beam dump, $8 \mathrm{~m}$ underground.

The primary physics goal of BDX is the search for light dark matter particles, produced by the primary electron beam in the target, by measuring their scattering on atomic electrons in the detector, resulting in a high-energy electromagnetic shower [39]. In particular, the experiment was designed and optimized considering the physics case of an invisibly decaying dark photon [40]. In the case of a negative result, $\mathrm{BDX}$ is expected to improve current exclusion limits by about 2 orders of magnitude. The experiment is also sensitive to long-lived particles decaying visibly to an $e^{+} e^{-}$or $\gamma \gamma$ pair, if the decay occurs within the detector volume. In this work, we derived the expected BDX sensitivity to a scalar particle considering, conservatively, an expected background contribution of five events, corresponding to a $95 \%$ C.L. sensitivity of $\sim 6$ signal events.

\section{COMPLEMENTARY PROBES}

\section{A. Anomalous magnetic moments}

One of the most sensitive bounds on light scalars for lepton-specific or muon-specific models is from the measurement of muon anomalous magnetic moment $a_{\mu} \equiv(g-2)_{\mu} / 2$. The current measured value of $a_{\mu}$ is larger than the SM prediction by [32]

$\Delta a_{\mu}=a_{\mu}(\mathrm{EXP})-a_{\mu}(\mathrm{SM})=(268 \pm 63 \pm 43) \times 10^{-11}$,

where the first and the second error bars indicate the experimental and theoretical systematical uncertainties, respectively. Introducing a new scalar that couples to muons can uplift the predicted value of $a_{\mu}$ by

$$
\Delta a_{\mu}(S)=\frac{g_{\mu}^{2}}{8 \pi^{2}} \int_{0}^{1} d z \frac{(1-z)^{2}(1+z)}{(1-z)^{2}+z\left(m_{S} / m_{\mu}\right)^{2}}
$$

and makes it agree with the current measured value. In the $g_{\mu}-m_{S}$ parameter space, we show the favored regions consistent with the measured value of $a_{\mu}$ as the shaded green region with the label $a_{\mu}( \pm 2 \sigma)$. We also report the excluded regions that yield a $5 \sigma$ discrepancy with respect to the measured value as the shaded gray region (joint with other exclusions) with the label $a_{\mu}(5 \sigma)$. Ongoing experimental and theoretical developments may reduce the experimental and theoretical systematical uncertainties by a factor of 4 and 2 , respectively, in the near future [22,34-36]. We indicated the favored and excluded regions for future $a_{\mu}$ measurements as the hashed green and gray regions, respectively.

\section{B. Cooling of SN 1987A}

A core-collapsed supernova ( $\mathrm{SN}$ ) behaves like a protoneutron star and cools mainly through neutrino diffusion under the standard SN model. The measured SN 1987A neutrino burst flux agrees with SN model predictions [41] and hence can be used to constrain light dark scalars, which may provide an extra cooling channel. For the extra cooling to be effective, dark scalars should be abundantly produced in the supernova and not decay or be trapped by nucleons and leptons along their exiting path. Reference [38] has considered an axionlike particle (ALP) with photon coupling $-\left(g_{\gamma \gamma} / 4\right) a F \tilde{F}$. The dominant production and trapping mechanisms are through a Primakoff process $\gamma N \rightarrow N S$ and an inverse-Primakoff process $S N \rightarrow N \gamma$, respectively. Given the production and trapping mechanisms for ALPs are identical to those of muon-specific dark scalars, we ignored the difference in the $C P$ quantum number and directly translated the resulting SN bounds on $g_{\gamma \gamma}$ from [38] to those on $g_{\mu}$ for muon-specific dark scalar through the relation

$$
g_{\gamma \gamma}=\frac{\alpha}{2 \pi}\left|\frac{g_{\mu}}{m_{\mu}} F_{1 / 2}\left(\frac{4 m_{\mu}^{2}}{m_{S}^{2}}\right)\right| .
$$

The translated bound is shown as the gray shaded region with label "SN 1987A" in the right panel of Fig. 7 [42]. Note that the exclusion region is cut off at $m_{S}=2 m_{\mu}$ : a heavier scalar would be always trapped in the supernova core, without contributing to the supernova cooling [7]. A similar analysis can be applied to the lepton-specific dark scalars except that the electron-dark scalar interaction should be also included for the trapping. Nevertheless, the resulting bound is weaker than the constraints of other electron beam-dump experiments for the parameter space of interest. Hence it is not explicitly labeled in the joint exclusion region in the left panel of Fig. 7. 

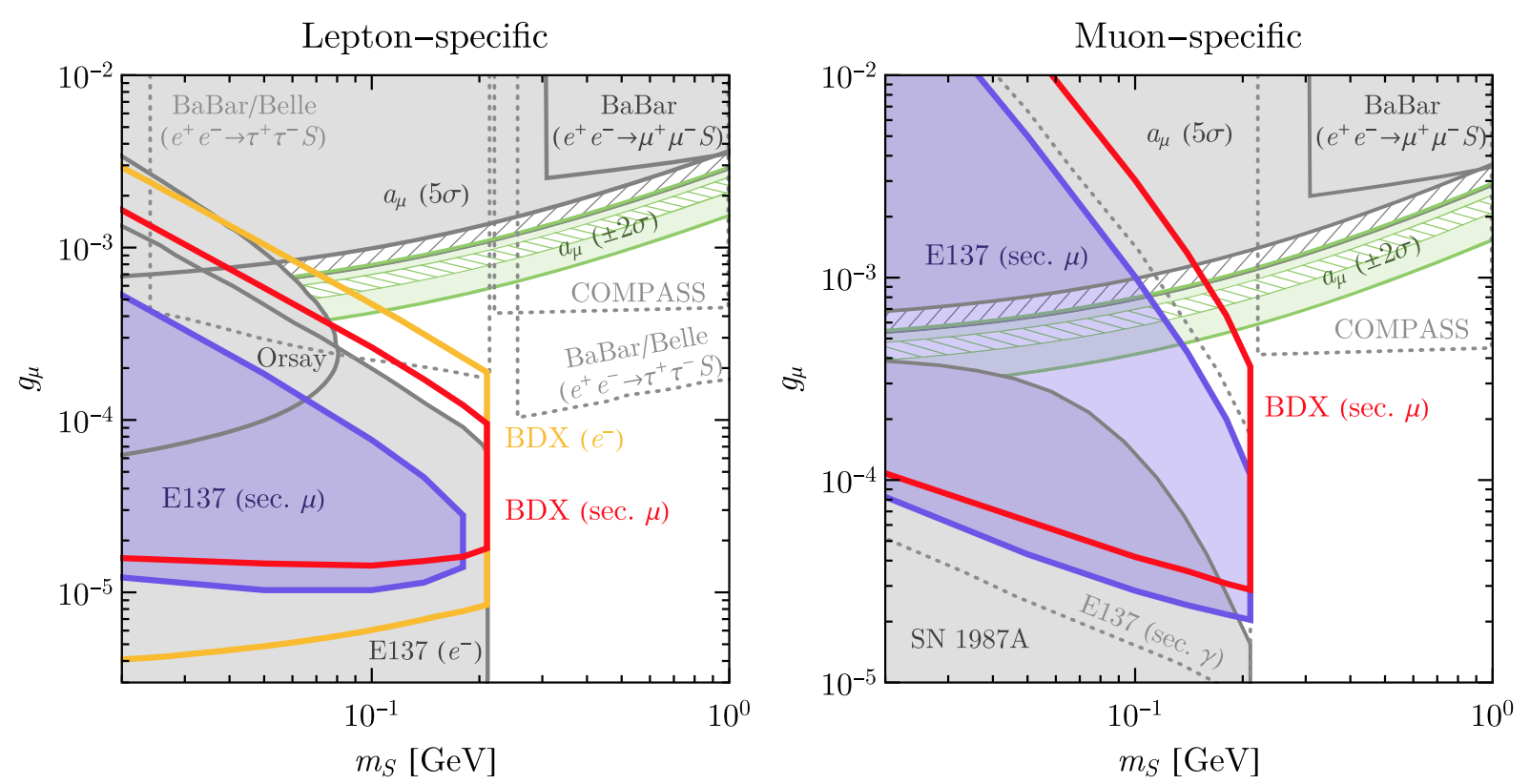

FIG. 7. 95\% C.L. exclusions and projections on dark scalar searches from secondary muons at E137 and BDX cross the $g_{\mu}-m_{S}$ plane, along with several other experimental projections and existing constraints. The left and right panels correspond to the lepton-specific model $\left(g_{e}: g_{\mu}: g_{\tau}=m_{e}: m_{\mu}: m_{\tau}\right)$ and muon-specific model $\left(g_{\mu} \neq 0, g_{e}=g_{\tau}=0\right)$, respectively. The blue regions represent constraints from secondary muons produced at E137. The red contours represent expected sensitivities from secondary muons produced at BDX. The yellow contour in the left panel represents the expected sensitivity through electron bremsstrahlung at BDX. The green regions show the $2 \sigma$-favored region from the current $a_{\mu}$ experiment [32]. The gray regions are combined exclusions based on existing experiments or observations. For the lepton-specific model, the gray region includes current $a_{\mu}$ measurement with $5 \sigma$ exclusions, BABAR limits based on $e^{+} e-\rightarrow \mu^{+} \mu^{-} S$ searches [33], and constraints from electron bremsstrahlung at Orsay and E137 [5]. For the muon-specific model, the gray region includes the same exclusions from $a_{\mu}$ and $B A B A R$ as the ones for the lepton-specific model. Besides it also includes constraints from SN 1987A (see Sec. VI B for more details). The hashed regions in both panels indicate the projected sensitivity of future $a_{\mu}$ measurements, assuming the current central value stays the same while the experimental and theoretical uncertainties will be improved by a factor of 4 and 2, respectively [22,34-36]. The dotted contour shows expected sensitivity if BABAR or Belle could search for $e^{+} e-\rightarrow \tau^{+} \tau^{-} S$ [5] or COMPASS could search for $\mu N \rightarrow \mu N S$ [37] with existing data. Note that we also indicate constraints from the secondary photons at E137 [38] as a dotted line given the uncertainties in the analysis as we discussed in Sec. VIC. Projected sensitivities for leptophilic dark scalars produced by future muon-beam experiments or secondary muons or photons at future protonbeam experiments can be found in $[6,12]$.

\section{Other fixed-target and beam-dump experiments}

In the case of the lepton-specific model, a constraint to $g_{\mu}$ can be derived considering the emission of scalar particles by $e^{+}$and $e^{-}$in the beam-induced electromagnetic shower. Even if the corresponding radiative cross section is suppressed by the factor $\left(m_{e} / m_{\mu}\right)^{2} \sim 2 \times 10^{-5}$ with respect to the muon case, the much larger track length of $e^{+}$and $e^{-}$ compensates for this, resulting in a non-negligible yield. In this case, since that the dump radiation length is much smaller than the target-detector distance, it is possible to assume that the scalar production happens always at the beginning of the target. This allows one to exclusion limit for scalars from the sensitivity to a visibly decaying dark photon $[43,44]$, by following the procedure depicted in [6]. In the left plot of Fig. 7, results from E137 and BDX are reported.

In the case of muon-specific model, there may be a significant contribution due to Primakoff process $\gamma N \rightarrow N S$ by bremsstrahlung photons in the beam dump. Although the $g_{\mu}$ contribution to the $g_{\gamma \gamma}$ is loop suppressed, a large flux of photons produced by the primary beam may still yield a large number of signal events and consequently a competitive bound on $g_{\mu}$. In particular, in Ref. [38] the original E137 bound on the mass and coupling of ALPs was cast to a limit on $g_{\gamma \gamma}$. In the calculation, authors used the photons track-length distribution reported in the original E137 paper [15] and evaluated the experimental acceptance only including the angular effects due to the opening angle between the ALP decay products. We note the following critical issues in this procedure. First, it ignores the contribution to the detector geometrical acceptance from the finite production angle of ALPs in the beam dump. Second, we found the photon track-length distribution reported in Ref. [15] to be inconsistent, in particular at low energy, with the result obtained from a FLUKA-based calculation that we performed (we cross-checked our result with the analytic predictions from showering theory $[45,46]$, finding an excellent agreement). We still report 
the result obtained translating the aforementioned limit on $g_{\gamma \gamma}$ to a limit on $g_{\mu}$, following the procedure depicted in Sec. VIB. We show the result as the gray dotted line with label "E137(sec. $\gamma$ )" on the right panel of Fig. 7, leaving a more detailed study for the future. Note that we explicitly checked the momentum squared of the $t$-channel photon from MC simulations and found it to be close to the onshell condition, thus allowing us to use Eq. (23).

\section{CONCLUSIONS}

Muons play a special role in the Standard Model of particles and elementary interactions. Some discrepancies between the observations and the SM predictions may be an indication of new physics. In particular, the coupling of muon to a new scalar particle $(S)$ could reconcile some of the tensions between data and model. In this paper we elaborated on the idea that an $\mathcal{O}(10) \mathrm{GeV}$ electron beam hitting the dump is a copious source of muons and electron beam-dump experiments have the ideal set up to detect the subsequent $S \rightarrow e^{+} e^{-}$and $S \rightarrow \gamma \gamma$ decays. A combination of simulation codes (FLUKA and GEANT4) has been used to generate, propagate, and project muon-produced scalars on E137 and BDX detectors. The numerical simulation realistically takes into account the different processes involved: muon production from the primary electron, resulting energy and angular spread, $S$ production, including production vertex distribution, $S$ propagation and decay product distribution within the detector volumes. Results obtained for the E137 setup extended the exclusion limits, consistently with the reported null results, to cover a broader area in $g_{\mu}$ vs $m_{S}$ parameter space. An even larger exclusion zone was obtained considering the planned BDX experiment. In conclusion, we demonstrated that electron beam-dump experiments (past and future) provide an enhanced sensitivity to new physics that may be specifically coupled not only to electrons or photons but also to muons.

\section{ACKNOWLEDGMENTS}

We thank Clive Fields for his help in determining the geometry and materials of the E137 experimental setup. A.C. and Y.Z. thank Oliver Mattelaer for his help in computing the cross section for the scalar radiative emission with MADGRAPH5_AMC@NLO 2.6.3.2. A.C. thanks Brian Batell and Rouven Essig for permission in reusing a figure from their E137 paper. Y.Z. thanks Brian Batell, Stefania Gori, Ahmed Ismail, and Felix Kahlhoefer for helpful discussions. M. B. and Y.Z. thank the Kavli Institute for Theoretical Physics (KITP) at University of California, Santa Barbara (UCSB) for hospitality, where their research was supported by the National Science Foundation under Grant No. NSF PHY1748958. Y. Z. also thanks the Aspen Center for Physics for hospitality, where his research is supported by National Science Foundation (NSF) Grant No. PHY-1607611. L. M. acknowledges support from Università degli Studi di Genova. Y.Z. acknowledges support from U.S. Department of Energy under Grant No. desc0015845.

\section{APPENDIX A: NUMERICAL COMPUTATION OF TOTAL CROSS SECTION AND DIFFERENTIAL DISTRIBUTIONS FOR THE SCALAR RADIATIVE EMISSION PROCESS}

The GEANT4-based code we developed to simulate radiative $S$ production by muons (see Appendix B) requires as input the total cross section $\sigma_{\mathrm{TOT}}$ for the process $\mu+N \rightarrow \mu+N+S$, as a function of muon kinetic energy, and the kinematic distribution of produced $S$ particles, as a function of scalar kinetic energy $T_{S}$ and production angle $\theta_{S}$. We computed numerically these quantities through the CALCHEP 3.7 [47] package. We implemented the aforementioned process in CALCHEP, by adding $S$ as a new particle, and including the corresponding interaction Lagrangian with muons, $\mathcal{L}=-g_{\ell} s \bar{\ell} \ell$ [see Eq. (1)]. The model was further modified by means of the USERFF.C function, in order to account for the combined atomic and nuclear form factor $G_{2}(t)$. We adopted the form-factor expression discussed in [48]. For most nuclei, $G_{2}$ is written as an incoherent sum of an elastic term and an inelastic contribution

$$
\begin{gathered}
G_{2}=G_{2}^{\mathrm{el}}+G_{2}^{\mathrm{inel}}, \\
G_{2}^{\mathrm{el}}(t)=Z^{2}\left(\frac{a^{2} t}{1+a^{2} t}\right)^{2}\left(\frac{1}{1+t / d}\right)^{2}, \\
G_{2}^{\mathrm{inel}}(t)=Z\left(\frac{a^{\prime 2} t}{1+a^{\prime 2} t}\right)^{2} \cdot W_{2}^{p}(t), \\
W_{2}^{p}(t)=\left(\frac{1+\tau\left(\mu_{p}^{2}-1\right)}{\left(1+\frac{t}{0.71 \mathrm{GeV}^{2}}\right)^{4}}\right)^{2},
\end{gathered}
$$

where $-t$ is the momentum-transferred squared, $\tau=\frac{t}{4 m_{p}^{2}}, Z$ and $A$ are the nuclei atomic number and mass, respectively, $a=113 Z^{-1 / 3} / m_{e}, d=0.164 \mathrm{GeV}^{2} A^{-2 / 3}, a^{\prime}=773 Z^{-2 / 3} / m_{e}$, $\mu_{p}=2.79$, and $m_{p}$ and $m_{e}$ are the proton and electron mass, respectively. For the specific case of hydrogen atom, we adopted the following form-factor parametrization [49,50]:

$$
\begin{aligned}
G_{2}^{\mathrm{el}}(t) & =(1-F(t))^{2} \cdot W_{2}^{p}(t), \\
G_{2}^{\text {inel }}(t) & =\left(1-|F(t)|^{2}\right) \cdot W_{2}^{p}(t), \\
F(t) & =\left(\frac{t}{4 \alpha^{2} m_{e}^{2}}+1\right)^{-2} .
\end{aligned}
$$

For each chemical element and $m_{S}$ value, we computed $\sigma_{\mathrm{TOT}}$ as a function of $E_{\mu}$ on a grid of 2000 points, from 


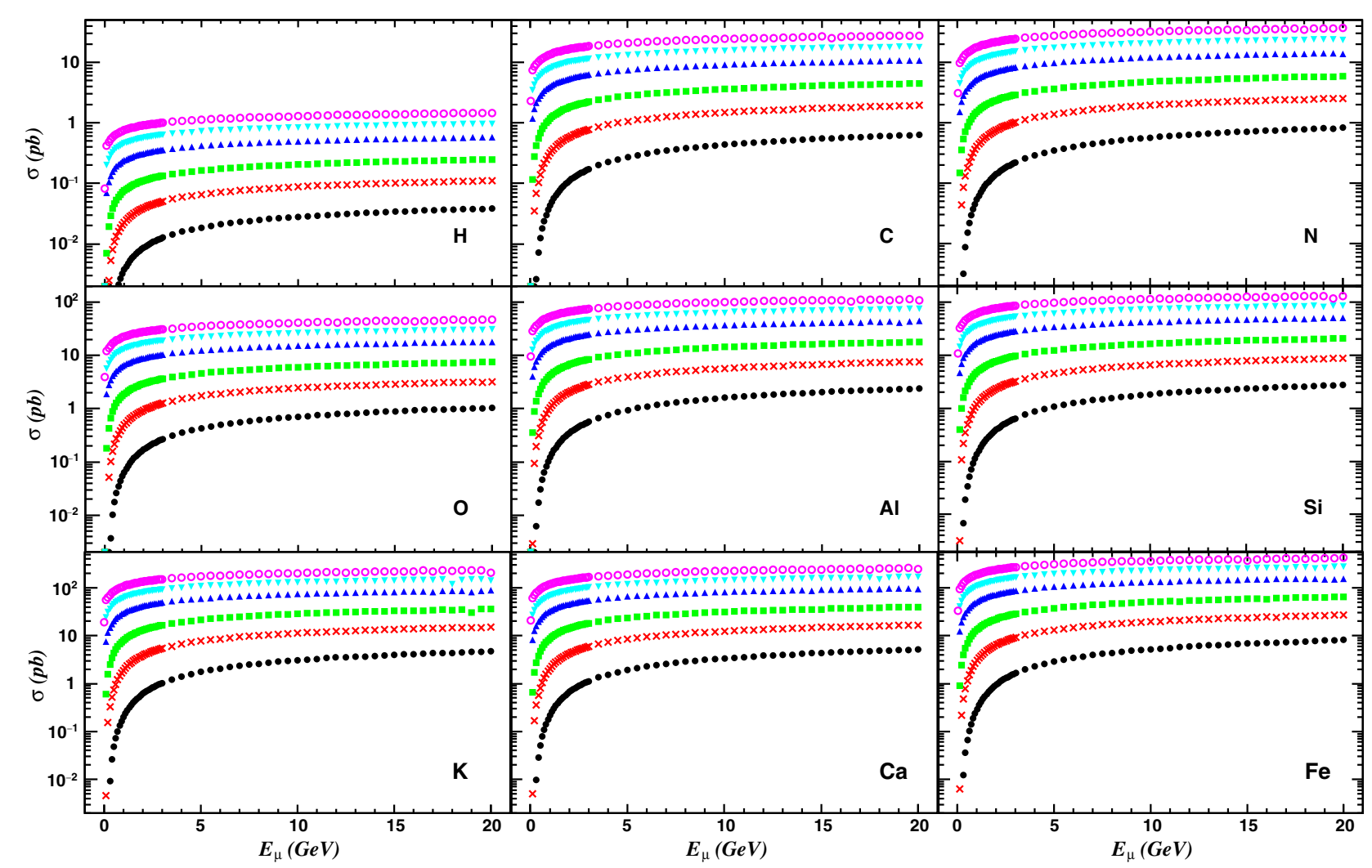

FIG. 8. Total cross section (in pb, $y$ axis) for the $\mu N \rightarrow \mu N S$ process as a function of the impinging muon kinetic energy (in GeV, $x$ axis), for $g_{\mu}=3.87 \times 10^{-4}$. Each plot refers to a specific material, as reported in the corresponding caption. Different colors refer to different values of the scalar mass: 210 (black full circles), 100 (red crosses), 50 (green squares), 20 (blue triangles), 10 (cyan triangles), and $5 \mathrm{MeV}$ (magenta open circles). Plots on the same line (column) share the same $y$-axis ( $x$-axis) scale.

$10 \mathrm{MeV}$ to $20 \mathrm{GeV}$. This interval corresponds to the energy range of interest in this work. The value of the coupling was fixed to $g_{\mu}^{0}=3.87 \times 10^{-4}$. The numerical integration was performed by first adapting the VEGAS grid with $i t_{1}=10$ runs, each with $N_{1}=100 \mathrm{k}$ iterations, and then performing the actual calculation through $i t_{2}=10$ runs, each with $N_{2}=100 \mathrm{k}$ iterations. The target accuracy was set to $1 \%$. For each point, we also generated $100 \times 10^{3}$ Monte Carlo events, that we used to sample the $T_{S}, \theta_{S}$ distribution. Figure 8 shows the total cross section for the $\mu+N \rightarrow \mu+N+S$ process, as a function of the impinging $\mu$ kinetic energy, for different values of the scalar mass and for the materials that are more relevant in the simulation.
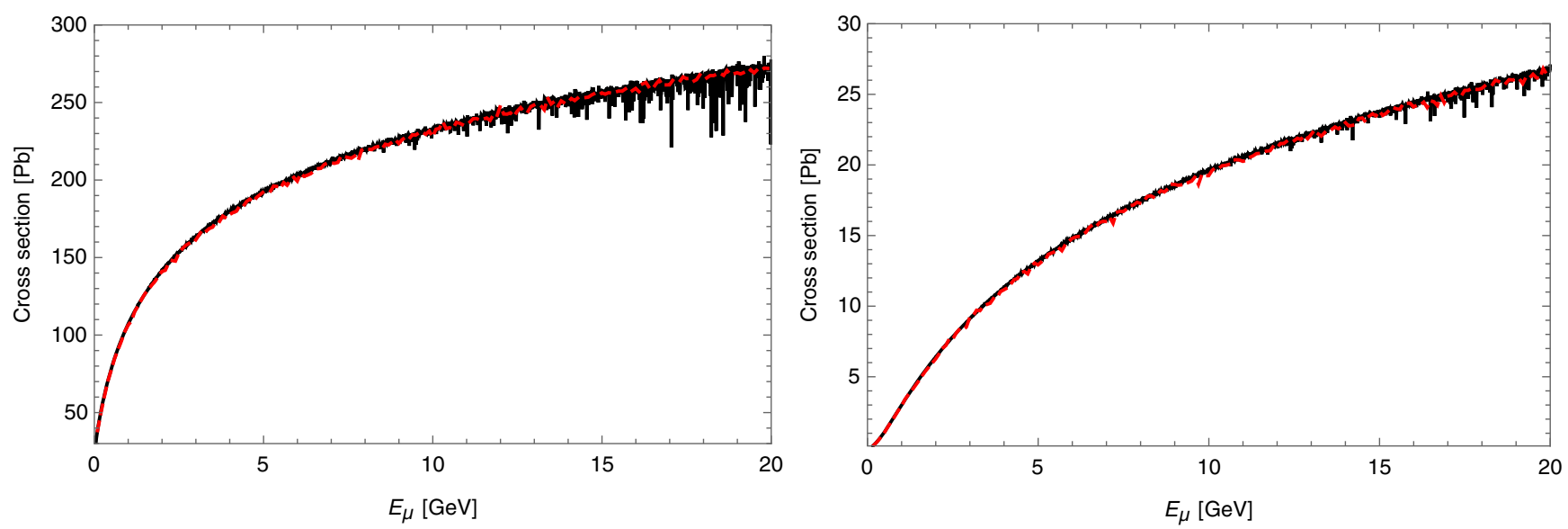

FIG. 9. Total cross section for the $\mu N \rightarrow \mu N S$ process for an iron target (for $g_{\mu}=3.87 \times 10^{-4}$ ), as a function of the impinging muon kinetic energy, as obtained from CALCHEP (continuous black line) and MG5 (dashed red line). The left plot is for $m_{S}=10 \mathrm{MeV}$, the right plot for $m_{S}=100 \mathrm{MeV}$. 
In order to confirm the numerical results, we compared them with those obtained from MADGRAPH5_AMC@NLO 2.6.3.2 [51] (MG5 for short in below), finding an excellent agreement for all materials and scalar masses [52]. As an example, Fig. 9 shows the comparison between the total cross section obtained from CALCHEP and MG5 for iron, as a function of the impinging muon kinetic energy, for the two values of the scalar mass $m_{S}=10 \mathrm{MeV}$ and $m_{S}=100 \mathrm{MeV}$.

\section{APPENDIX B: GEANT IMPLEMENTATION OF SCALAR RADIATIVE EMISSION PROCESS}

We implemented the scalar radiative emission process by muons in GEANT4 as follows. A new class G4SCALAR, inheriting from G4PARTICLEDEFINITION, was introduced to describe the scalar particle $S$. The class contains a singleton G4PARTICLEDEFINITION instance, that effectively introduces the new particle in the simulation. The singleton is instantiated through a static method with a single parameter, the mass of the scalar particle. This allows one to set $m_{S}$ dynamically at the simulation start, in order to perform the multistep numerical evaluation of the event yield, as described in Sec. IV.

The production process was implemented through the new class G4MUONSCALARPRODUCTION, inheriting from G4VDiscreteProcess and implementing the following mother class virtual methods:

(i) GetMeanFreePATH, that returns the mean free path associated to the $S$ production by a muon with given energy, in a given material;

(ii) PostStepDoIt, that specifies the actions to perform when a new scalar is produced, instructing the GEANT4application to create a new scalar particle at the interaction vertex with given momentum, sampled from the differential cross section, and to alter the impinging muon momentum to ensure total four-momentum conservation.
By making use of a class inheriting from G4VDiscreteProcess, and adding it to the list of allowed physics processes for muons in the main physics list, the GEANT4 kernel automatically handles the $S$ production coherently with the other muons interaction and propagation mechanisms.

The mean free path $\lambda$ for a muon with kinetic energy $T_{\mu}$ propagating in a certain chemical element is computed from the relation $\lambda^{-1}=n \sigma_{\mathrm{TOT}}$, with $n$ being the number of atomic nuclei per unit volume. The value of $n$ is obtained from the internal GEANT4database, while $\sigma_{\text {TOT }}$ is loaded by the G4MUONSCALARPRODUCTION class at creation, from precomputed data-more details are given in Appendix A. In the case of compounds, $\lambda$ is obtained as an average:

$$
\lambda^{-1}=\sum_{i} n_{i} \sigma_{\mathrm{TOT}}^{i}
$$

with the index " $i$ " running over the chemical elements forming the compound and $\sigma_{\mathrm{TOT}}^{i}, n_{i}$ being the corresponding cross section and atomic number density.

To describe the emission of the new scalar particle, first the particle kinetic energy $T_{S}$ and polar angle $\theta_{S}$ with respect to the impinging muon direction are sampled from a precomputed distribution, for the corresponding $E_{\mu}$ value and chemical element. The azimuthal angle $\phi_{S}$ is generated assuming a flat distribution. This allows one to define the corresponding four-momentum $P_{S}$. The impinging muon four-momentum is thus altered to enforce four-momentum conservation, $P_{\mu}^{\prime}=P_{\mu}-P_{S}$ (this assumes that the target nucleus stays at rest during the interaction). In the case of compounds, the chemical element through which the impinging muon interacts is randomly selected from those forming the element, associating to each a statistical weight $w_{i}=n_{i} \sigma_{\text {TOT }}^{i}$. Then, the same procedure described before is adopted.
[1] G. W. Bennett et al. (Muon (g-2) Collaboration), Phys. Rev. Lett. 92, 161802 (2004).

[2] M. Battaglieri et al., arXiv:1707.04591.

[3] G. Krnjaic, Phys. Rev. D 94, 073009 (2016).

[4] C.-Y. Chen, H. Davoudiasl, W. J. Marciano, and C. Zhang, Phys. Rev. D 93, 035006 (2016).

[5] B. Batell, N. Lange, D. McKeen, M. Pospelov, and A. Ritz, Phys. Rev. D 95, 075003 (2017).

[6] C.-Y. Chen, M. Pospelov, and Y.-M. Zhong, Phys. Rev. D 95, 115005 (2017).

[7] B. Batell, A. Freitas, A. Ismail, and D. McKeen, Phys. Rev. D 98, 055026 (2018).
[8] G. D’Ambrosio, G. F. Giudice, G. Isidori, and A. Strumia, Nucl. Phys. B645, 155 (2002).

[9] To be more precise, it is an antimuon beam generated through proton beam. In the rest of the paper we indicate both $\mu^{-}$and $\mu^{+}$as "muon" or $\mu$ for simplicity.

[10] Y. Kahn, G. Krnjaic, N. Tran, and A. Whitbeck, J. High Energy Phys. 09 (2018) 153.

[11] C.-Y. Chen, J. Kozaczuk, and Y.-M. Zhong, J. High Energy Phys. 10 (2018) 154.

[12] A. Berlin, S. Gori, P. Schuster, and N. Toro, Phys. Rev. D 98, 035011 (2018). 
[13] E. Cortina Gil et al. (NA62 Collaboration), J. Instrum. 12, P05025 (2017).

[14] W. M. Bonivento, J. Phys. Conf. Ser. 878, 012014 (2017).

[15] J. D. Bjorken, S. Ecklund, W. R. Nelson, A. Abashian, C. Church, B. Lu, L. W. Mo, T. A. Nunamaker, and P. Rassmann, Phys. Rev. D 38, 3375 (1988).

[16] M. Bondí (BDX Collaboration), EPJ Web Conf. 142, 01005 (2017).

[17] Y.-S. Liu, D. McKeen, and G. A. Miller, Phys. Rev. D 95, 036010 (2017).

[18] J. Cox, F. Martin, M. L. Perl, T. H. Tan, W. T. Toner, T. F. Zipf, and W. L. Lakin, Nucl. Instrum. Methods Phys. Res., Sect. A 69, 77 (1969).

[19] W. Nelson, Nucl. Instrum. Methods Phys. Res., Sect. A 66, 293 (1968).

[20] T. Bhlen, F. Cerutti, M. Chin, A. Fassó, A. Ferrari, P. Ortega, A. Mairani, P. Sala, G. Smirnov, and V. Vlachoudis, Nucl. Data Sheets 120, 211 (2014).

[21] A. Ferrari, P. R. Sala, A. Fasso, and J. Ranft, Technical Report Nos. CERN-2005-010, No. SLAC-R-773, No. INFN-TC-05-11, 2005.

[22] A. Chapelain (Muon g-2 Collaboration), EPJ Web Conf. 137, 08001 (2017).

[23] B. Batell, R. Essig, and Z. Surujon, Phys. Rev. Lett. 113, 171802 (2014).

[24] C. Patrignani et al. (Particle Data Group), Chin. Phys. C 40, 100001 (2016).

[25] A. Chilton, Health Phys. 34, 715 (1978).

[26] S. Agostinelli et al., Nucl. Instrum. Methods Phys. Res., Sect. A 506, 250 (2003).

[27] D. R. Walz, H. P. Noyes, and R. L. Carezani, Phys. Rev. A 29, 2110 (1984).

[28] D. R. Walz, SLAC Technical Report No. SLAC-TN-67-031, SLAC, 1967.

[29] A. Seryi and T. O. Raubenheimer, SLAC Technical Report No. SLAC-PUB-8595, SLAC, 2000.

[30] A. Freyberger (CEBAF Commissioning Team), in Proceedings of 6th International Particle Accelerator Conference (IPAC 2015), Richmond, Virginia, USA, 2015 (JACoW, Geneva,Switzerland, 2015).

[31] M. Wiseman, C. K. Sinclair, R. Whitney, M. Zarecky, and R. Vetterlein, in Proceedings of the 1997 Particle Accelerator Conference (Cat. No. 97CH36167) (IEEE, 1997), Vol. 3, pp. 3761-3763.
[32] C. Patrignani et al. (Particle Data Group), Chin. Phys. C 40, 100001 (2016).

[33] J. P. Lees et al. (BABAR Collaboration), Phys. Rev. D 94, 011102 (2016).

[34] J. Grange et al. (Muon g-2 Collaboration), arXiv:1501 .06858 .

[35] T. Mibe (J-PARC g-2 Collaboration), Chin. Phys. C 34, 745 (2010).

[36] F. Jegerlehner, Acta Phys. Pol. B 49, 1157 (2018).

[37] R. Essig, R. Harnik, J. Kaplan, and N. Toro, Phys. Rev. D 82, 113008 (2010).

[38] M. J. Dolan, T. Ferber, C. Hearty, F. Kahlhoefer, and K. Schmidt-Hoberg, J. High Energy Phys. 12 (2017) 094.

[39] M. Battaglieri et al. (BDX Collaboration), arXiv:1607 .01390 .

[40] E. Izaguirre, G. Krnjaic, P. Schuster, and N. Toro, Phys. Rev. D 88, 114015 (2013).

[41] G. G. Raffelt, Stars as Laboratories for Fundamental Physics (University of Chicago Press, 1996).

[42] The SN bound for the muon-specific dark scalar here is a factor of 2 lower than those in Fig. 7. We assumed $g_{\gamma \gamma}$ for the scalar and the pseudoscalar to be identical while Fig. 7 assumed the two couplings to differ by a factor of 2 .

[43] B. Holdom, Phys. Lett. 166B, 196 (1986).

[44] S. Andreas, C. Niebuhr, and A. Ringwald, Phys. Rev. D 86, 095019 (2012).

[45] B. Rossi and K. Greisen, Rev. Mod. Phys. 13, 240 (1941).

[46] G. Clement and P. Kessler, Nuovo Cimento 37, 876 (1965).

[47] A. Belyaev, N. D. Christensen, and A. Pukhov, Comput. Phys. Commun. 184, 1729 (2013).

[48] J. D. Bjorken, R. Essig, P. Schuster, and N. Toro, Phys. Rev. D 80, 075018 (2009).

[49] K. J. Kim and Y.-S. Tsai, Phys. Rev. D 8, 3109 (1973).

[50] Y.-S. Tsai, Rev. Mod. Phys. 46, 815 (1974); 49, 421 (1977).

[51] J. Alwall, R. Frederix, S. Frixione, V. Hirschi, F. Maltoni, O. Mattelaer, H. S. Shao, T. Stelzer, P. Torrielli, and M. Zaro, J. High Energy Phys. 07 (2014) 079.

[52] The computation of the cross section for the scalar radiative emission process with MADGRAPH5_AMC@NLO 2.6.3.2 required an ad hoc fix to the matrix-element computation, to prevent a nonphysical discontinuity in the momentum transferred distribution. 